\title{
PULSAR OBSERVATIONS AT DECAMETER WAVELENGTHS USING THE GAURIBIDANUR TELESCOPE
}

\author{
A. A. Deshpande and V. Radhakrishnan \\ Raman Research Institute
}

\begin{abstract}
We report measurement of pulse profiles at $34.5 \mathrm{MHz}$ of 8 pulsars using the Decameter-wave Radio Telescope at Gauribidanur, India. The dispersion measures of these pulsars range from 3 to 35 $\mathrm{pc} \mathrm{cm}^{-3}$. None of the pulsars show any significant interpulse emission at this frequency which conflicts with earlier claims from 25- $\mathrm{MHz}$ observations (Bruck and Ustimenko 1976, Bruck and Ustimenko $1977 \mathrm{a}$, Bruck and Ustimenko 1979). The intrinsic pulse width of PSR 1919+21 at $34.5 \mathrm{MHz}$ appears to be smaller than at higher frequencies. These observations were made during the period 1984-86 and were reported by Deshpande (1987).
\end{abstract}

\section{Introduction}

The behavior of pulsars at decametric wavelengths is still poorly known because of observational difficulties due to both the propagation effects in the intervening interstellar medium and the high galactic background at frequencies below $100 \mathrm{MHz}$. The limited data from low frequency observations (Bruck 1987, Kuz'min 1986, Shitov, Malofeev, and Izvekova 1988) have already shown that such observations are valuable. We report here observations of pulsars using the Decameter-wave Radio Telescope at Gauribidanur, India, operating at $34.5 \mathrm{MHz}$.

\section{Observations}

In our observations, the sensitivity of the telescope (originally a transit instrument) was improved by incorporating a tracking system (Deshpande, Shevgaonkar, and Sastry 1989) to enable observation over $42 x^{\prime \prime}(\delta)$ minutes in a day. Observations were made using two different methods. 1. Observations of strong but not highly dispersed pulsar signals were attempted at first. A single-frequency-channel receiver with a $30-\mathrm{kHz}$ bandwidth (optimum for low dispersion measures) was used. Observations were made in the directions of 20 pulsars during December 1984 to February 1985. A stringent detection criterion was used for increasing the reliability of detection. For this purpose, the raw data were folded over two-pulse period stretches and were tested for significant detection of two pulses separated exactly by one period. With this criterion, 8 pulsars were detected successfully and average pulse profiles, estimates for the average pulse energy and other parameters were obtained for them.

2. Subsequently, a scheme was devised to enable high time-resolution observations of highly dispersed pulsar signals. This scheme involves a basic swept-frequency dedispersion procedure (Sutton et al. 1970). A programmable sweeping local oscillator system was built (Deshpande 1990) and used with the existing 128-channel autocorrelation receiver (Shankar and Shankar 1990) to obtain intensity patterns with high resolution in the frequency domain. To avoid the need for accurate gain calibration of individual frequency channels, as well as the need for absolute synchronization of the sweep, the sweep reset period was deliberately chosen to be slightly different from the apparent period for a given pulsar. The intensity pattern across the frequency band then drifts slowly, sampling the pulsar signal in different longitude ranges. The patterns were sampled frequently and combined later by compensating for this drift. In this way higher resolution can be obtained for strong pulsar signals. This set-up allowed observations of pulsar signals that are highly dispersed at our frequency.

Details of the observations and data analysis will be published elsewhere.

\section{Results}

The main results can be summarized as follows.

1. Average pulse profiles were measured for 8 pulsars and reliable estimates of average pulse energies were obtained (table 1). Typical pulse profiles are displayed in figure 1 . Three of the pulsars in table 1 are detected for the first time at a decametric wavelength.

2. With the exception of PSR 0943+10, all the observed pulsars show turnovers in their spectra at frequencies above $35 \mathrm{MHz}$.

3. None of the 8 pulsars show any significant interpulse emission at our frequency. The $3 \sigma$ limits are: $2 \%$ of the main pulse energy for PSR $0834+06$, $6 \%$ for PSR $0950+08,3.5 \%$ for PSR $1133+16$ and 

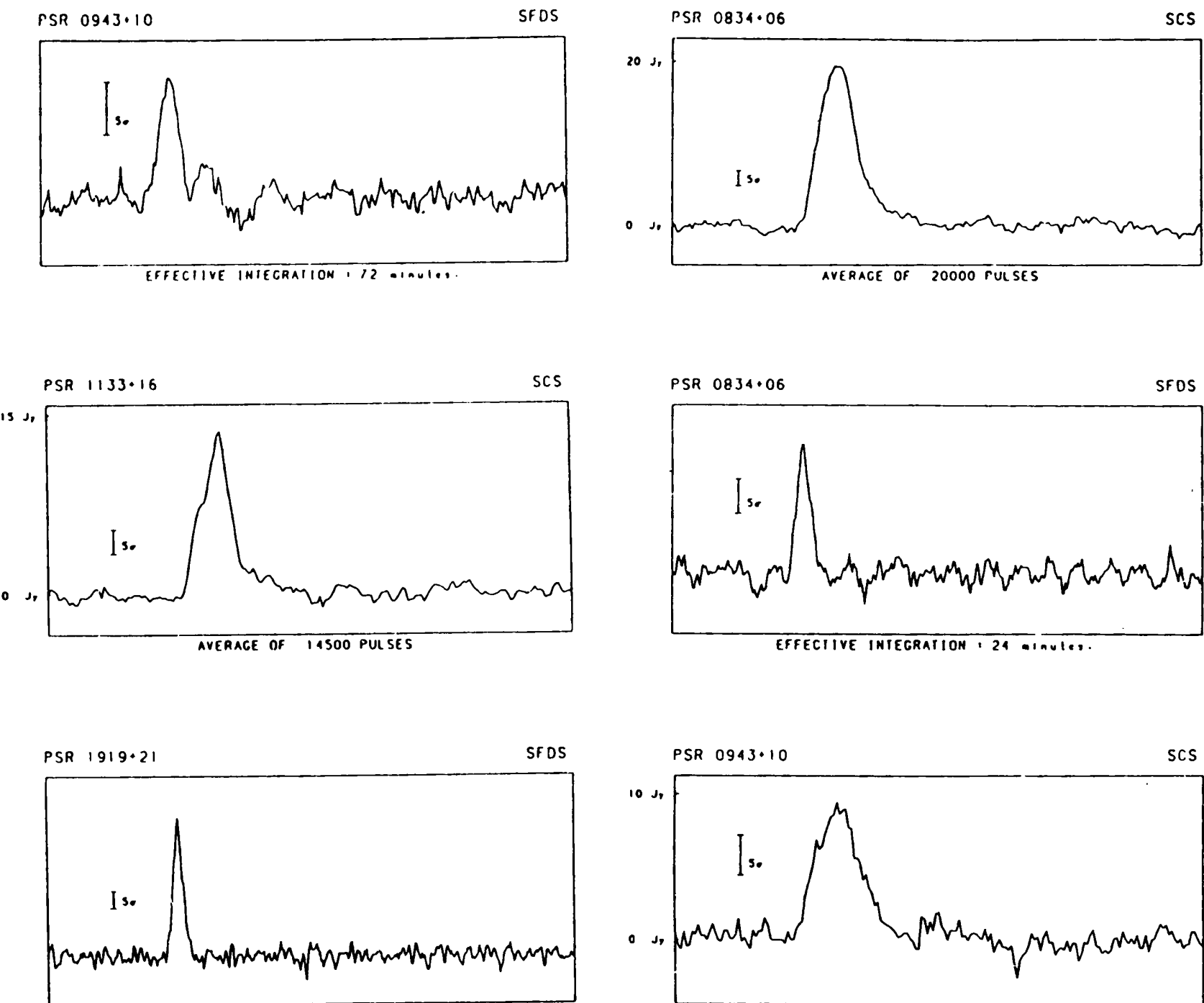

EFFECIIVE INIEGRATION II

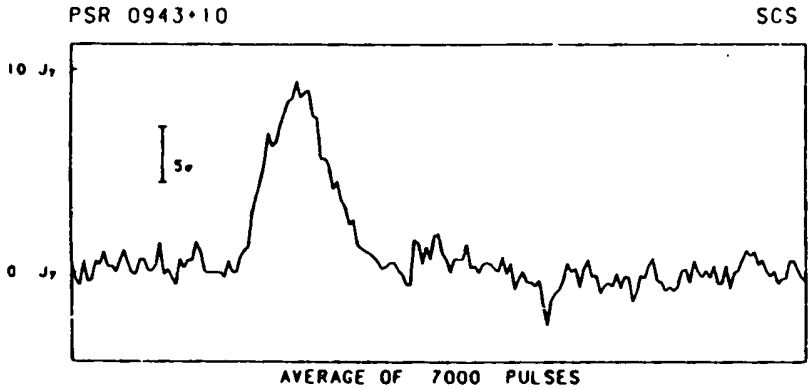

Figure 1 Pulse profiles at $34.5 \mathrm{MHz}$. The code at the top right corner indicates the method of observation. SCS = Single Frequency Channel Scheme. SFDS = Swept Frequency Dedispersion Scheme.

Table 1 Average pulse energy at $34.5 \mathrm{MHz}$

\begin{tabular}{|lr|}
\hline Pulsar & $\begin{array}{r}\text { Average pulse energy } \\
\left(10^{-20} \mathrm{~J} \mathrm{~m}^{-2} \mathrm{~Hz}^{-1}\right)\end{array}$ \\
\hline PSR 0628-28 & $1750 \pm 480$ \\
PSR 0809+74 & $710 \pm 320$ \\
PSR 0834+06 & $2100 \pm 100$ \\
PSR 0942-13 & $110 \pm 60$ \\
PSR 0943+10 & \\
PSR 0950+08 & $960 \pm 120$ \\
PSR 1133+16 & $420 \pm 60$ \\
PSR 1919+21 & $900 \pm 90$ \\
\hline
\end{tabular}

†ew detections at decametric wavelengths

$\$$ Errors quoted are $5 \sigma$ values.

$6 \%$ for PSR $0943+10$. Unless the spectrum of the interpulse emission is much steeper than that of the main pulse, these observations conflict with the re- sults reported by Bruck and Ustimenko $(1976,1977$, 1979). Recent 25-MHz observations at Arecibo (Phillips and Wolszczan 1989) have also not detected any significant interpulse emission.

4. The pulse widths measured at $34.5 \mathrm{MHz}$ indicate that they cannot be predicted from measurements at higher frequencies using a single scaling law for all pulsars (as seen in similar earlier observations, e.g. Kuz'min et al. 1986). In fact, for PSR 1919+21 the intrinsic width at $34.5 \mathrm{MHz}$ appears to be smaller than that at higher frequencies.

5. The above result implies that it is difficult to predict the intrinsic pulse shape at low frequencies. Therefore, scatter broadening cannot be reliably estimated from the observed pulse shapes. Measurements of the decorrelation bandwidth (of interstellar scintillations) appear to be more reliable for estimating scatter broadening. However, at low frequencies these are too small to be measured easily. 